\title{
THE GRAVITATIONAL LENSING IN REDSHIFT-SPACE CORRELATION FUNCTIONS OF GALAXIES AND QUASARS
}

\author{
Takahiko Matsubara ${ }^{1,2}$ \\ Department of Physics and Astronomy, The Johns Hopkins University, 3400 N.Charles Street, \\ Baltimore, MD 21218 \\ matsu@pha.jhu.edu
}

\begin{abstract}
The gravitational lensing, as well as the velocity field and the cosmological light-cone warp, changes the observed correlation function of high-redshift objects. We present an analytical expression of 3D correlation function, simultaneously including those three effects. When two objects are separated over several hundreds Mpc along a line of sight, the observed correlation function is dominated by the effect of gravitational lensing rather than the intrinsic clustering. For a canonical lambda-CDM model, the lensing signals in the galaxy-galaxy and galaxy-QSO correlations are beyond noise levels in large-scale redshift surveys like the Sloan Digital Sky Survey.
\end{abstract}

Subject headings: cosmology: theory — gravitational lensing — large-scale structure of universe - methods: statistical

\section{INTRODUCTION}

The correlation function is one of the fundamental quantities in searching into the physical origin of the universe. In large-scale redshift surveys, redshifts and spherical positions on the sky of luminous objects are used for estimating the spatial distribution of mass, but the former is distorted by inhomogeneity of the universe.

Two intrinsic distortion effect on the correlation function originate in the velocity field (Kaiser 1987) and the cosmological warp (Ballinger, Peacock \& Heavens 1996; Matsubara \& Suto 1996; Matsubara 2000). The former comes from the fact that an observed redshift corresponds to the recession velocity which is composed not only of the expansion of the universe but also of peculiar velocities. The latter distortion is brought about by the nonlinear mapping of the objects from the expanding curved space on a light cone onto a flat redshift space (Alcock \& Paczyński 1979).

\footnotetext{
${ }^{1}$ Department of Physics, The University of Tokyo, Hongo 7-3-1, Tokyo 113-0033, Japan

${ }^{2}$ Research Center for the Early Universe, Faculty of Science, The University of Tokyo, Tokyo 113-0033, Japan
} 
In addition to those intrinsic distortions, there are secondary distortions which are due to perturbations of light rays. While the redshift is altered by the Sachs-Wolfe effect (Sachs \& Wolfe 1967), the spherical position is recast by the gravitational lensing (Schneider et al. 1992; Mellier 1999; Bartelmann \& Schneider 1999). Although the Sachs-Wolfe effect is not so important, the gravitational lensing can affect the observable correlation function in forthcoming redshift surveys as this effect is efficient for high-redshift objects (Gunn 1967).

The effect of the gravitational lensing on the angular functions $w(\theta)$ have been intensively investigated so far (Bartelmann \& Schneider 1999; Moessner et al. 1998; Kaiser 1992; Villumsen 1996). Recently, among others, cross correlations of galaxies at different redshifts (Moessner et al. 1998) are successfully applied to the commissioning data from the Sloan Digital Sky Survey (Jain

et al. 2000), to single out the weak lensing effect.

As for the 3D correlation function in redshift space, a qualitative treatment of the lensing effect is given in Suto et al. (1999) to estimate the upper limit of the effect, using the phenomenological Dyer-Roeder distance (Dyer \& Roeder 1973). It is still not clear whether or not the lensing is actually efficient where the intrinsic correlation function is negligible on scales comparable to $100 \mathrm{Mpc}$. The main purpose of this letter is to give a quantitative treatment of this issue, consistently including velocity and cosmological distortions, and consequently to show the weak lensing actually has detectable effects on 3D correlation function in redshift space when large-scale redshift surveys like Sloan Digital Sky Survey (SDSS) are available.

\section{OBSERVABLE QUANTITIES}

We take the homogeneous, isotropic FRW metric with scalar perturbations in longitudinal gauge:

$$
d s^{2}=a^{2}(\tau)\left\{-(1+2 \phi) d \tau^{2}+(1-2 \phi)\left[d \chi^{2}+S_{K}^{2}(\chi)\left(d \theta^{2}+\sin ^{2} \theta d \varphi^{2}\right)\right]\right\},
$$

where $a$ is the scale factor, and $\tau$ is the conformal time, $d \tau=d t / a$, and $S_{K}$ is the comoving angular distance of the spatial curvature $K=\Omega_{0}+\lambda_{0}-1$. For example, $S_{K}(\chi)=(-K)^{-1 / 2} \sinh \left[(-K)^{1 / 2} \chi\right]$ for open universe, $K<0$, and $S_{K}(\chi)=\chi$ for flat universe, $K=0$. We adopt the unit $c=H_{0}=1$ throughout this letter.

From the first-order Einstein equation of the metric in equation (1), the density contrast $\delta(\boldsymbol{x}, \tau)$ and the velocity field $v^{i}(\boldsymbol{x}, \tau)$ on scales much less than the curvature scale satisfy

$$
\triangle \phi=\frac{3 \Omega_{0}}{2} \frac{\delta}{a}, \quad v^{i}=\frac{2}{3 \Omega_{0}} a H f \nabla^{i} \phi
$$

where $H=\dot{a} / a, f=d \ln D / d \ln a$, and $D$ is the linear growth rate (Peebles 1980). The Laplacian $\triangle$ is taken with respect to comoving coordinates.

Let us consider a light ray emitted from an object at comoving coordinates $(\chi, \theta, \varphi ; \tau)$, which an observer recieves at $\tau_{0}$. The conventional redshift $z=a^{-1}-1$ is given by $\chi=\int_{0}^{z} d z H^{-1}$, but 
the actually observed redshift is changed by the line-of-sight peculiar velocity, $V=n_{i} v^{i}$, where $n_{i}$ is a line-of-sight unit vector, and also by the gravitational potential, $\phi$. From the time-component of the geodesic equation of the light ray, the observed redshift $z_{\mathrm{s}}$ is given by (Sachs \& Wolfe 1967)

$$
z_{\mathrm{s}}=z+(1+z)\left[V(\chi)-V(0)-\phi(\chi)+\phi(0)-2 \int_{\tau_{0}-\chi}^{\tau_{0}} d \tau \frac{\partial \phi}{\partial \tau}\right]
$$

where we abbreviate the function on a light cone as $V(\chi) \equiv V\left(\chi, \theta, \varphi ; \tau_{0}-\chi\right)$, and so as $\phi(\chi)$. The integral is performed on the light cone for a fixed direction of line of sight.

Now, we consider the small angle approximation so that light rays are confined to a narrow cone around the polar axis, $\theta \ll 1$, and introduce new coordinates $\theta_{1}=\theta \cos \varphi, \theta_{2}=\theta \sin \varphi$, following Kaiser (1998). Adopting the Born approximation, the angular components of the geodesic equation reduces to equation for the observed angular components $\theta_{\mathrm{s} a}(a=1,2)$ :

$$
\theta_{\mathrm{s} a}=\theta_{a}+\frac{2}{S_{K}(\chi)} \int_{0}^{\chi} d \chi^{\prime} S_{K}\left(\chi-\chi^{\prime}\right) \partial_{a} \phi\left(\chi^{\prime}\right)
$$

where $\partial_{a}=S_{K}{ }^{-1} \partial / \partial \theta_{a}$.

The apparent luminosity of the light is magnified by a factor $A(z)=\left|\operatorname{det}\left(\partial \theta_{\mathrm{s} b} / \partial \theta_{a}\right)\right|=1+2 \kappa$, where $\kappa$ is a local convergence field of weak lensing (Schneider et al. 1992; Bernardeau et al. 1997; Kaiser 1998) for a fixed redshift of source object:

$$
\kappa(z, \boldsymbol{\theta})=\int_{0}^{\chi} d \chi^{\prime} g\left(\chi, \chi^{\prime}\right) \partial_{a} \partial_{a} \phi\left(\chi^{\prime}\right) ; \quad g\left(\chi, \chi^{\prime}\right) \equiv \frac{S_{K}\left(\chi^{\prime}\right) S_{K}\left(\chi-\chi^{\prime}\right)}{S_{K}(\chi)} .
$$

Due to the magnification, the observed apparent magnitude $m_{\mathrm{s}}$ is given by $m_{\mathrm{s}}=m-2.5 \log _{10} A=$ $m-5 \kappa / \ln 10$ (Broadhurst et al. 1995), where $m$ is the apparent magnitude in the absence of lensing.

The magnitude-limited number density in real space $n_{\mathrm{r}}(z, \boldsymbol{\theta} ;<m)$ and that in observed redshift space $n_{\mathrm{s}}\left(z_{\mathrm{s}}, \boldsymbol{\theta}_{\mathrm{s}} ;<m_{\mathrm{s}}\right)$ are related by the number conservation equation, $n_{\mathrm{r}} z^{2} d z d^{2} \theta=n_{\mathrm{s}} z_{\mathrm{s}}^{2} d z_{\mathrm{s}} d^{2} \theta_{\mathrm{s}}$, while the number density is given by $n(z, \boldsymbol{\theta} ;<m)=[1+\delta(z, \boldsymbol{\theta})] N(z ;<m) /\left(4 \pi z^{2}\right)$, where $\delta(z, \boldsymbol{\theta})$ is the density contrast and $N(z ;<m)$ is the magnitude-limited number count per redshift. Evaluating the Jacobian, one obtains the relation between $n$ and $n_{\mathrm{s}}$, as well as the relation between density contrasts. The result contains the terms with $\delta, V, \phi$ and their derivatives. For fluctuations on a scale $k$ in units of Hubble distance, such variables scales as $V \sim k^{-1} \delta, \partial V \sim \delta, \phi \sim k^{-2} \delta$, $\partial \phi \sim k^{-1} \delta$, and $\partial^{2} \phi \sim \delta$, where $\partial$ schematically represents the spatial derivatives in comoving coordinates. Consistently to the small angle approximation, we neglect the fluctuations which scale as $k^{-1}$ and $k^{-2}$, because $k$ is large enough on scales below Hubble distance. Eventually, the distorted density contrast is given by

$$
\delta_{\mathrm{s}}=\delta_{\mathrm{r}}-\frac{1+z}{H} \frac{\partial V}{\partial \chi}+(5 \alpha-2) \kappa
$$

where $\delta_{\mathrm{r}}(z, \boldsymbol{\theta})$ is the number density contrast of the objects in real space, and $H(z)$ is the Hubble parameter at $z$. The logarithmic slope of the number counts $\alpha$ at the limiting magnitude $m$ is given 
by $\alpha(z, m)=\partial \log _{10} N(z ;<m) / \partial m$ (c.f., Moessner, Jain \& Villumsen 1998). The first two terms of equation (6) depends strongly on radius $z$ and angles $\boldsymbol{\theta}$, while the last, the lens surface density, depends strongly on angles but very weakly on radius. The first term of equation (6) is the real density fluctuations, the second term is the velocity distortion (Kaiser 1987; Matsubara \& Suto 1996), and the last term is the effect of weak lensing, which consists of the contribution from the modulation by magnification bias, $5 \alpha \kappa$, and of the alternation of surface density by lensing, $-2 \kappa$. In the following, we denote each term in equation (6) as $\delta_{\mathrm{s}}=\delta_{\mathrm{r}}+\delta_{\mathrm{v}}+\delta_{\mathrm{l}}$.

\section{CORRELATION FUNCTION}

We consider the correlation function between two objects $\left(z_{1}, \boldsymbol{\theta}_{1}\right)$ and $\left(z_{2}, \boldsymbol{\theta}_{2}\right)$ in the small angle approximation, $\theta \equiv\left|\boldsymbol{\theta}_{1}-\boldsymbol{\theta}_{2}\right| \ll 1$, and assume $z_{1} \leq z_{2}$ without loss of generality. In the absence of the lensing term $\delta_{1}$, the correlation function is given by Matsubara \& Suto (1996), which generalize the work by Hamilton (1992) to high-redshift objects. In the coordinate system $\left(z_{1}, z_{2}, \theta\right)$, their result, with slight modification allowing difference of the kind of two objects, is expressed as follows:

$$
\begin{aligned}
\xi_{\mathrm{MS}}=\langle & {\left.\left[\delta_{\mathrm{r}}(1)+\delta_{\mathrm{v}}(1)\right]\left[\delta_{\mathrm{r}}(2)+\delta_{\mathrm{v}}(2)\right]\right\rangle } \\
= & {\left[1+\frac{1}{3}\left(\beta_{1}+\beta_{2}\right)+\frac{1}{5} \beta_{1} \beta_{2}\right] \xi_{0}(x ; \bar{z}) P_{0}(\mu) } \\
& \quad-\left[\frac{2}{3}\left(\beta_{1}+\beta_{2}\right)+\frac{4}{7} \beta_{1} \beta_{2}\right] \xi_{2}(x ; \bar{z}) P_{2}(\mu)+\frac{8}{35} \beta_{1} \beta_{2} \xi_{4}(x ; \bar{z}) P_{4}(\mu),
\end{aligned}
$$

where $\bar{z}=\left(z_{1}+z_{2}\right) / 2, \beta_{i}=f\left(z_{i}\right) / b_{i}\left(z_{i}\right) \simeq \Omega^{0.6}\left(z_{i}\right) / b_{i}\left(z_{i}\right)$, and $b_{i}\left(z_{i}\right)$ is the bias parameter of object $i(i=1,2)$ at redshift $z_{i}$. Similarly, a bar for any variable means the evaluation at $\bar{z}$ and subscripts 1,2 assume evaluation at objects 1 and 2, respectively. We denote the comoving

separation $x \equiv \sqrt{\left[S_{K}(\bar{\chi})\right]^{2} \theta^{2}+\left(\chi_{2}-\chi_{1}\right)^{2}}$, the comoving cosine $\mu \equiv\left(\chi_{2}-\chi_{1}\right) / x$, and $P_{n}$ 's are Legendre polynomials, and

$$
\xi_{2 l}(x ; \bar{z})=\bar{b}^{2} \int_{0}^{\infty} \frac{k^{2} d k}{2 \pi^{2}} j_{2 l}(k x) P(k ; \bar{z}),
$$

where $P(k ; z)$ is the power spectrum at redshift $z$. This formula is valid only for distant observer approximation, $x \ll \chi_{1}$ and $\theta \ll 1$. We have omitted the finger-of-God effect which is only important on scales less than $10 h^{-1} \mathrm{Mpc}$ or $1000 \mathrm{~km} / \mathrm{s}$. In the following, we are interested in the scales of $30 \mathrm{Mpc}$ or larger where lensing effect appears, so that we can safely ignore the nonlinear effect like finger-of-God effect.

Adopting the small angle approximation (e.g., Bernardeau et al. 1997; Kaiser 1998; Moessner et al. 1998), the correlations involving the lensing term $\delta_{\mathrm{l}}$ are obtained as

$$
\begin{aligned}
& \xi_{\mathrm{rl}}=\left\langle\delta_{\mathrm{r}}(1) \delta_{\mathrm{l}}(2)\right\rangle=\frac{3}{2} \Omega_{0} b_{1}\left(5 \alpha_{2}-2\right) g\left(\chi_{2}, \chi_{1}\right)\left(1+z_{1}\right) \xi_{\mathrm{p}}\left[\theta S_{K}\left(\chi_{1}\right) ; z_{1}\right], \\
& \xi_{\mathrm{ll}}=\left\langle\delta_{\mathrm{l}}(1) \delta_{\mathrm{l}}(2)\right\rangle=\left(\frac{3}{2} \Omega_{0}\right)^{2}\left(5 \alpha_{1}-2\right)\left(5 \alpha_{2}-2\right)
\end{aligned}
$$




$$
\times \int_{0}^{\chi_{1}} d \chi(z) g\left(\chi_{1}, \chi\right) g\left(\chi_{2}, \chi\right)(1+z)^{2} \xi_{\mathrm{p}}\left[\theta S_{K}(\chi) ; z\right]
$$

where $d \chi(z)=d z / H(z), \xi_{\mathrm{p}}$ is the projected correlation function defined by

$$
\xi_{\mathrm{p}}(y ; z)=\int_{-\infty}^{\infty} d x \xi\left(\sqrt{x^{2}+y^{2}} ; z\right)=\int_{0}^{\infty} \frac{k d k}{2 \pi} J_{0}(k y) P(k ; z),
$$

and $\xi(r ; z)=\xi_{0}(r ; z)$ is the correlation function in real space at $z$. The terms $\left\langle\delta_{1}(1) \delta_{\mathrm{r}}(2)\right\rangle$, $\left\langle\delta_{\mathrm{l}}(1) \delta_{\mathrm{r}}(2)\right\rangle$ are zero for $z_{1} \leq z_{2}$ in the small angle approximation. The velocity-lensing term, $\xi_{\mathrm{vl}}=\left\langle\delta_{\mathrm{v}}(1) \delta_{\mathrm{l}}(2)\right\rangle$ is explicitly calculated to be zero, which is because the term $\delta_{\mathrm{v}}$ only depends on fluctuations along the line of sight which are smoothed out. If we convolve the above expression of $\xi_{\mathrm{rl}}$ and $\xi_{\mathrm{ll}}$ with a selection function along the line of sight, we obtain the form of angular correlation function with the effect of weak lensing (Bartelmann 1995; Villumsen 1996; Dolag \& Bartelmann 1997; Moessner et al. 1998; Moessner \& Jain 1998).

Thus the total correlation function in 3D redshift space is given by $\xi_{\text {tot }}\left(z_{1}, z_{2}, \theta\right)=\xi_{\mathrm{MS}}+\xi_{\mathrm{rl}}+\xi_{\mathrm{ll}}$ for $z_{1} \leq z_{2}$ and $\theta \ll 1$. The first term dominates on scales much smaller than Hubble distance, while the last two terms dominate on scales comparable to the Hubble distance along the line of sight. Therefore, even though $\xi_{\mathrm{MS}}$ is valid only for $z_{1}-z_{2} \ll z_{1}$ (distant observer approximation), we can use the form $\xi_{\text {tot }}$ even when $z_{1}-z_{2} \sim z_{1}$.

In Figure 1, we plot the total correlation function $\xi_{\text {tot }}$ together with each component, $\xi_{\mathrm{MS}}$, $\xi_{\mathrm{rl}}$, and $\xi_{\mathrm{ll}}$. With the choice of the CDM-like initial power spectrum (Bardeen et al. 1986) with a shape parameter $\Gamma=0.25$ and a linear amplitude $\sigma_{8}=1$, we use the fitting formula for the fully non-linear power spectrum of Peacock \& Dodds (1996) for lensing correlations, $\xi_{\mathrm{rl}}$ and $\xi_{\mathrm{ll}}$. Linear predictions are also plotted in the lower panels. The nonlinearity of the intrinsic correlation $\xi_{\mathrm{MS}}$, which is only important for the region $z_{2}-z_{1} \lesssim 0.003$, is ignored. We exemplify the low-density flat model with $\Omega_{0}=0.3, \lambda=0.7$. In the upper panels, the slope of the number counts is assumed as $\alpha=1$, and the bias factor as $b=1$, regardless of the redshift. This example corresponds to $z \sim 0.2$ and $m \sim 18$ of galaxies as seen in Table 1, in which the slope $\alpha$ is calculated from the B-band luminosity function of APM galaxies (Loveday et al. 1992), and of quasars (Boyle et al. 1988). The limiting magnitudes assumed in Table 1 correspond to estimated SDSS redshift data of galaxies and quasars. In practice, the slope $\alpha$ can be observationally determined for individual catalogue of quasar or galaxy redshift surveys. Each component of correlations roughly scales as $\xi_{\mathrm{MS}} \propto \sigma_{8}^{2} b_{1} b_{2}, \xi_{\mathrm{rl}} \propto \sigma_{8}^{2} \Omega_{0} b_{1}\left(5 \alpha_{2}-2\right), \xi_{\mathrm{ll}} \propto \sigma_{8}{ }^{2} \Omega_{0}^{2}\left(5 \alpha_{1}-2\right)\left(5 \alpha_{2}-2\right)$ for other parameters and models.

In the lower panels of Figure 1, the galaxy-galaxy (G-G), galaxy-QSO (G-Q), and QSO-QSO (Q-Q) correlations are plotted, assuming the SDSS slope $\alpha$ of Table 1 . The bias parameter is set $b=1$ and 3 for galaxies and quasars, respectively. The line-of-sight separations are large enough in lower panels, so that the intrinsic clustering is negligible. 


\section{DISCUSSION}

The absolute value of the intrinsic clustering component $\xi_{\mathrm{MS}}$ is a decreasing function of the line-of-sight separation, $z_{2}-z_{1}$, except the vicinity of zero crossings. On the other hand, the lens-lens component $\xi_{11}$ is almost independent on the separation and the density-lens component $\xi_{\mathrm{rl}}$ is an increasing function. Those behaviors are understood by the fact that the weak lensing is efficient between the object and the observer. Thus, intrinsic clustering component dominates for small separations, while lens-lens and/or density-lens components dominates for large separations along the direction of line of sight.

The lower panels in Figure 1 show the region where lensing contribution dominates in the case of the SDSS magnitude limits to illustrate the typical magnitude of correlations. Are those lensing signals detectable? The statistical uncertainty in estimating the correlation function is given by $(\delta \xi)^{2}=\Omega /\left(\delta \Omega N_{1} N_{2}\right)$ (Peebles 1980), where $N_{1}$ and $N_{2}$ are numbers of object in the bin used for redshifts $z_{1}$ and $z_{2}$, respectively, $\Omega$ is the solid angle subtended by the survey area, and $\delta \Omega$ is the fraction in the bin used for angle $\theta$. To increase the signal to noise ratio, it is desirable to use large bins for $\theta$. To be specific, we consider a bin $\left[1^{\prime}, 10^{\prime}\right]$, and theoretical curves are integrated accordingly, so that $\delta \Omega \sim 100 \pi\left[\operatorname{arcmin}^{2}\right]$. The effective scale of this bin is given by $\theta_{\text {eff }}=10^{\prime} / \sqrt{2}$ for $\xi \propto \theta^{-1}$. In the SDSS, $\Omega \sim \pi[\operatorname{str}] \sim 1.2 \pi \times 10^{7}\left[\operatorname{arcmin}^{2}\right]$, and the estimated numbers of galaxies and quasars are $N_{\mathrm{G}}=10^{6}$ and $N_{\mathrm{Q}}=1.7 \times 10^{5}$, respectively. Assuming we take sufficiently large bins of redshifts (this choice is similar to considering angular correlation functions), the consequent estimates of the statistical error are given by $5.0 \times 10^{-4}$ for G-G, $8.5 \times 10^{-4}$ for G-Q, and $2.9 \times 10^{-3}$ for $\mathrm{Q}-\mathrm{Q}$ correlations, which are plotted in lower panels. The $\mathrm{S} / \mathrm{N}$ ratios turn out to be about 10 , 1.3 , and 0.22 for G-G, G-Q, and Q-Q correlations, respectively.

Therefore, the weak lensing in 3D correlation function of galaxies in the SDSS is definitely detectable, and the detection of galaxy-QSO cross-correlation is marginal, while the quasar correlation by lensing is below the noise level in the SDSS. In order to detect the QSO-QSO lensing effect, the sample should be at least 5 times larger than the SDSS, or parameters $\sigma_{8}, \Omega_{0}, b, \alpha$ should be larger than assumed values.

In summary, we have obtained a theoretical prediction of correlation function in redshift space, taking into account the effect of weak lensing, together with velocity distortions and cosmological distortions on a light-cone. Each effect contributes differently to the correlation function, and is realistically detectable. Our result provides a fundamental link between theoretical models and the observed correlation function in the 3D redshift survey data. Besides the determination of the power spectrum itself, various cosmological parameters, especially the bias parameter, can be estimated by proper likelihood analyses, including KL transform of the correlation matrix (Vogeley \& Szalay 1996; Matsubara et al. 2000). One may also be tempted to assume the cosmological parameters before analysing data. In which case, the error originated in choosing the wrong cosmological model is roughly given by the order of the redshift $z$ times the error of cosmological parameters, since the Alcock-Paczyński effect is roughly proportional to $z$ up to $z=1-2$. 
I am grateful to Bhuvnesh Jain for many helpful discussions. I would like to thank Yasushi

Suto and Alex Szalay for stimulating discussions. I acknowledge support from JSPS Postdoctoral Fellowships for Research Abroad.

\section{REFERENCES}

Alcock, C. \& Paczyński, B. 1979, Nature, 281, 358

Ballinger, W. E. \& Peacock, J. A. \& Heavens, A. F. 1996, MNRAS, 282, 877

Bardeen, J. M., Bond, J. R., Kaiser, N. \& Szalay, A. S. 1986, ApJ, 304, 15

Bartelmann, M. 1995, A\&A, 298, 661

Bartelmann, M. \& Schneider, P. 1999, to be submitted to Physics Reports (astro-ph/9912508)

Bernardeau, F., van Waerbeke, L. \& Mellier, Y. 1997, A\&A, 322, 1

Boyle, B. J., Shanks, T., Peterson, B. A. 1988, MNRAS, 235, 935

Broadhurst, T. J., Taylor, A. N. \& Peacock, J. A. 1995, ApJ, 438, 49

Dolag, K. \& Bartelmann, M.1997, MNRAS, 291, 446

Dyer, C. C. \& Roeder, R. C. 1973, ApJ, 180, L31

Gunn, J. E. 1967, ApJ, 147, 61

Hamilton, A. J. S. 1992, ApJ, 385, L5

Jain, B. et al. 2000, in preparation

Kaiser, N. 1987, MNRAS, 227, 1

Kaiser, N. 1992, ApJ, 388, 272

Kaiser, N. 1998, ApJ, 498, 26

Loveday, J., Peterson, B. A., Efstathiou, G. \& Maddox, S. J. 1992, ApJ, 390, 338

Matsubara, T. 2000, ApJ, in press (astro-ph/9908056)

Matsubara, T. \& Suto, Y. 1996, ApJ, 460, 51

Matsubara, T., Szalay, A. S. \& Landy, S. D. 1999, to appear in ApJL (astro-ph/9911151)

Mellier, Y. 1999, ARA\&A, 37, 127

Moessner, R. \& Jain, B. 1998, MNRAS, 294, L18 
Moessner, R., Jain, B. \& Villumsen, J. V. 1998, MNRAS, 294, 291

Peacock, J. A. \& Dodds, S. J. 1996, MNRAS, 280, L19

Peebles, P. J. E. 1980, The Large-Scale Structure of the Universe (Princeton; Princeton University Press)

Sachs, R. W. \& Wolfe, A. M. 1967, ApJ, 147, 73

Schneider, P., Ehlers, J., Falco,. E. E. 1992, Gravitational Lenses, (Springer)

Suto, Y., Magira, H., Jing, Y. P., Matsubara, T. \& Yamamoto, K. 1999, Prog. Theor. Phys. Suppl., 133,183

Villumsen, J. V. 1996, MNRAS, 281, 369

Vogeley, M. S. \& Szalay, A. S. 1996, ApJ, 465, 34

This preprint was prepared with the AAS $\mathrm{LAT}_{\mathrm{E}} \mathrm{X}$ macros v5.0. 

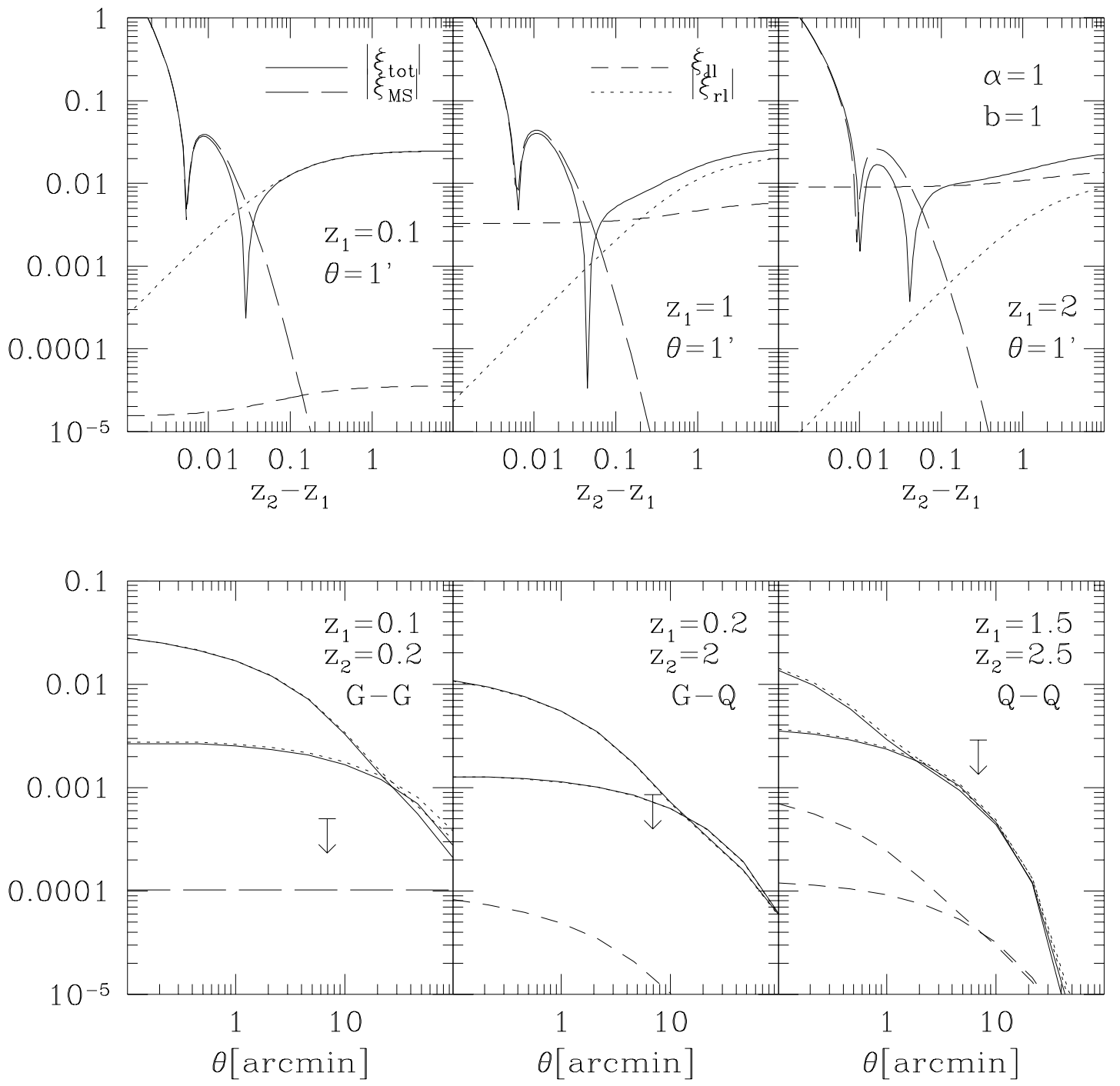

Fig. 1. - The correlation function along the line of sight for a flat LCDM model. Long-dashed lines: intrinsic clustering, $\xi_{\mathrm{MS}}$, dashed lines: lens-lens correlation, $\xi_{11}$, dotted lines: density-lens correlation, $\xi_{\mathrm{rl}}$, solid lines: total correlation function, $\xi_{\text {tot }}$. In the upper panels, $z_{1}$ and $\theta$ are fixed in each panel, with $\alpha=1, b=1$. In lower panels, $z_{1}$ and $z_{2}$ are fixed and parameters which mimic the SDSS redshift catalogue (see text) are assumed. The noise levels for the SDSS are also shown. From left to right are plotted galaxy-galaxy, galaxy-QSO, and QSO-QSO correlations. Nonlinear predictions are plotted except the lines which are not enhanced on small angles in lower panels. 
Table 1. Slope $\alpha(z, m)$ of the number counts with fixed redshifts.

\begin{tabular}{ccccccc|cccccc}
\hline \hline \multicolumn{1}{c}{$\operatorname{Galaxies}^{\mathrm{a}}\left(B_{\lim }=18.8\right)$} & \multicolumn{6}{c}{$\operatorname{Quasars}^{\mathrm{b}}\left(B_{\lim }=20.0\right)$} \\
\hline$z \ldots$ & 0.05 & 0.10 & 0.15 & 0.20 & 0.25 & 0.30 & 0.5 & 1.0 & 1.5 & 2.0 & 2.5 & 3.0 \\
$\alpha \ldots$ & 0.20 & 0.42 & 0.75 & 1.2 & 1.8 & 2.6 & 0.26 & 0.29 & 0.30 & 0.29 & 0.29 & 0.28 \\
\hline
\end{tabular}

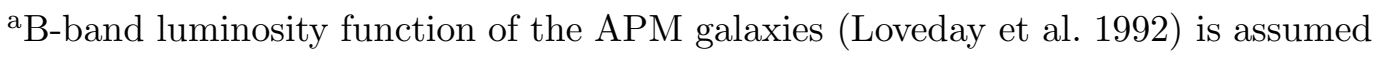

${ }^{\mathrm{b}}$ B-band luminosity function of the quasar sample (Boyle et al. 1988) is assumed 